Variations tibétaines, Et autres...

\title{
SINOR Denis (17 avril 1916- 12 janvier 2011)
}

\section{Françoise Aubin}

\section{OpenEdition \\ Journals}

\section{Édition électronique}

URL : https://journals.openedition.org/emscat/1948

DOI : 10.4000/emscat.1948

ISSN : 2101-0013

\section{Éditeur}

Centre d'Etudes Mongoles \& Sibériennes / École Pratique des Hautes Études

\section{Référence électronique}

Françoise Aubin, «SINor Denis (17 avril 1916- 12 janvier 2011) », Études mongoles et sibériennes,

centrasiatiques et tibétaines [En ligne], 42 | 2011, mis en ligne le 20 décembre 2011, consulté le 13 juillet 2021. URL : http://journals.openedition.org/emscat/1948 ; DOI : https://doi.org/10.4000/emscat.1948

Ce document a été généré automatiquement le 13 juillet 2021.

(c) Tous droits réservés 


\title{
SINOR Denis (17 avril 1916- 12 janvier 2011)
}

\author{
Françoise Aubin
}

1 L'émotion qu'a suscitée dans le monde orientaliste l'annonce de la disparition de Denis Sinor, le 12 janvier 2011, est à la mesure de la personnalité et de l'ampleur de l'action du disparu: il avait 95 ans, mais il semblait immortel. Les turcologues, les mongolisants, les linguistes du monde altaïque, les historiens de l'Asie intérieure et bien d'autres encore échangeaient des mails émus, comme s'ils se sentaient soudain devenir orphelins. La trace laissée par Denis Sinor peut s'évaluer à l'aune de ses nombreuses réalisations.

2 Il a d'abord été un auteur et conférencier fécond. À chaque intervention qu'il annonçait avant un colloque ou un volume collectif, quelque minime qu'en fut le sujet, on pouvait être sûr d'y faire toujours des découvertes ${ }^{1}$. Ce pouvait être des considérations générales dans le domaine de la linguistique altaïque et de la parenté ouralo-altaïque, comme on le voit dans un recueil reprenant en 1990 ses articles précédents, Essays in Comparative Altaic Linguistics (Indiana Univ.). Bien souvent la comparaison s'exerçait de manière jouissive sur un terme particulier qu'il retournait en tous sens avec une érudition étourdissante : je me souviens particulièrement de l'immensité du savoir qu'il avait déployé à partir d'un terme chinois hebo 合缺 ou hebao 合包, déjà traité par le $\mathrm{P}$. Henry Serruys, et autour duquel il avait brodé sur les récipients, les cercueils et les cucurbitacées (Acta Orientalia Hungarica - cité infra AOH -, XLVIII/3, 1995, pp. 457-464); ou encore sur les transports par voie d'eau en Eurasie centrale (article de 1961, repris dans Essays in Comparative Altaic Linguistics : 156-179).

Mais ce pouvait être aussi des fresques d'histoire eurasiatique médiévale, toujours renouvelées, sobres et brèves. Ainsi une contribution nouvelle en son temps sur chevaux et pâturage dans l'histoire de l'Asie intérieure (Oriens Extremus, 19/1-2, 1972, pp. 171-183, repris comme Asian Studies, Reprint Series, Research Institute, Bloomington, 18); ou la présence turco-mongole dans le monde méditerranéen et pontique à l'époque pré-ottomane (AOH, XXXVI/1-3, 1983, pp. 485-501). Le plus beau cadeau qu'il fit à ses collègues présents et futurs fut son Introduction à l'étude de l'Eurasie centrale 
(Wiesbaden : Otto Harrassowitz, 1963), dans lequel il livrait d'une manière pédagogique toujours valable d'immenses connaissances bibliographiques en toutes langues. Mais, au fait, n'est-ce pas lui qui a vulgarisé les concepts d'« Asie intérieure ", "Inner Asia » et « Eurasie centrale » (distincts de l'« Asie centrale » russophone), notamment grâce à son manuel d'initiation si commode pour les débutants (Inner Asia: History, Civilization, Languages. A Syllabus, Indiana Univ. \& Mouton 1969, réimpression Curzon 1997) ${ }^{2}$ ?

Et à partir des années 1960, tout son incroyable dynamisme s'est investi dans le développement et l'affermissement des études sur l'Asie intérieure. Il y eut d'abord la PIAC, Permanent International Altaistic Conference ${ }^{3}$, fondée au $24^{e}$ Congrès international des Orientalistes à Munich en 1957 : j'y étais et je pense même être le dernier témoin survivant de cet événement qui ne souleva alors pas l'enthousiasme de ses rares témoins. L'idée émanait de trois orientalistes de premier plan : Annemarie von Gabain, Walther Heissig et Omeljan Pritsak. L'aventure paraissait folle, comme le confessa plus tard Sinor, qui n'y croyait guère : réunir sur un mode informel et plaisant, tous les ans en un pays différent, si possible une fois à l'Ouest, une fois à l'Est, des spécialistes de tout âge venus du monde entier pour parler d'une quelconque question touchant à la théorie altaïque et à l'Asie intérieure. Le 4 e colloque se tint à Cambridge, où Sinor était alors en poste, et depuis lors il ne cessa d'en être le secrétaire général et l'animateur jusqu'à l'âge de 91 ans en 2007. Et si, en 2011, cette PIAC sur laquelle on pariait peu à ses débuts, atteint sa $54^{\mathrm{e}}$ session, il est certain qu'on le doit, en grande partie, au charisme de Sinor. Elle se tient justement à Bloomington, dans l'université d'Indiana qui fut celle de Sinor durant presque un demi-siècle, et son objet devait être la célébration du $95^{\mathrm{e}}$ anniversaire du patriarche.

5 Sa vie scientifique et universitaire se divise en deux parts: d'abord les années de formation et de découverte de la voie à suivre; puis à partir de 1962, l'ancrage à l'Université d'Indiana qu'il a puissamment contribué à élever au rang de capitale des études comparatives centre-asiatiques et altaïques. Il commence en effet par créer là un Department of Uralic and Altaic Studies (dénommé maintenant Department of Central Eurasian Studies), qu'il préside de 1963 à 1981 - il prend sa retraite d'enseignant en 1986 ; et dépendant de ce département, il fonde en 1967 un prestigieux Asian Studies Research Institute qui s'est développé jusqu'à donner naissance à plusieurs centres ou unités de recherche: Inner Asian \& Uralic National Ressource Center (IAUNRC), Sinor Research Institute for Inner Asian Studies (SRIFIAS), Center for Languages of the Central Asian Region (CeLCAR), enfin un Summer Language Workshop (SWSEEL). En 1967 encore, il lance un périodique, le Journal of Asian History (ayant atteint actuellement le $\mathrm{n}^{\circ} 44$, toujours chez Otto Harrassowitz à Wiesbaden), pour suppléer, dit-il, à l'absence d'une revue d'histoire destinée autant aux historiens non orientalistes qu'aux spécialistes de chacune des régions asiatiques (à l'exception du Moyen-Orient antique), et il en reste le directeur jusqu'en 2008, bien au-delà de sa quatre-vingt-dixième année. Simultanément les honneurs et les responsabilités glorieuses pleuvent sur lui, ainsi à l'UNESCO, à l'Encyclopaedia Britannica ou à la tête de la série des Uralic and Altaic Series. Ses diverses activités universitaires et scientifico-mondaines à partir des années soixante sont bien connues et on peut en trouver l'énoncé sur Internet, par exemple sur le site www.indiana.edu/ ceus/faculty/sinor.shtml. Je ne m'y attarderai donc pas, choisissant de privilégier la personnalité de Denis plutôt que les institutions. Les débuts de notre amitié, avec échange régulier de lettres et de tirés à part, remontent à 1969 ; et, de 1976 à 2008, j'ai été une des heureuses bénéficiaires des trente et une lettres annuelles de vœux qu'il a envoyées urbi et orbi, y narrant les événements de sa vie durant les douze 
mois écoulés. En un recto-verso d'une page, il livrait un délicieux récit plein d'humour - lecture très attendue de fin d'année et plaisante re-lecture en cours d'année. On voudrait les citer toutes, ces trente et une lettres, et il vaudrait la peine de les publier. Le trait de la vie de Sinor qui frappe le plus est son goût pour les voyages, soit les voyages exotiques au loin, bien qu'il détestât les aéroports et les avions, soit des traversées de l'Amérique du Nord, au volant d'une puissante voiture ou plus souvent d'une moto (jusqu'en 2006), les milliers de kilomètres s'ajoutant aux milliers de kilomètres, pour sa plus grande joie : « As much as I hate the killing airports as much I love long-distance driving in the Great American Emptiness that lies between the Mississipi and California ».

61982 est l'année où son goût pour les défis au destin apparaît le plus crûment. Il est alors en congé sabbatique et de nombreux voyages sont prévus. « The year started with a bang, quite literally, a bang in my heart which, since my last heart-attack in 1975, seemed to have been content with the statu quo, imposing difficult but not unbearable conditions on my life. Now things changed radically, and for about six months there barely was a night without at least one cardiac attack ». Son état est alors tel que le seul remède possible est l'opération: les examens de fin avril révèlent en effet cinq obstructions des artères coronaires. Et que croyez-vous qu'il fit? Déjà, début avril, il avait répondu à une invitation de l'Académie tadjike et participé à un colloque à Dushanbe, passé une semaine à Moscou et une autre à Londres. Puis, fin mai, on le retrouve à Budapest où il a, comme à l'accoutumée, quantité d'affaires à régler, et bientôt à Uppsala pour la $25^{\mathrm{e}}$ PIAC où il est réélu secrétaire général pour cinq ans; il prend ensuite le train de Copenhague à Lausanne pour se remémorer là les souvenirs de son enfance et, dans la foulée, il participe à une rencontre de l'UNESCO à Paris et travaille intensément durant deux jours à la Bibliothèque nationale. "By that time I lived on a dozen or more 'emergency pills' a day and could barely walk a few steps without severe chest pains ». Il racontait, bien plus tard, qu'il se revoyait assis place du Palais-Royal, cloué par la douleur au point de ne pouvoir même faire signe à l'ami qui l'attendait assis à la terrasse d'un café voisin. Cependant il passe encore un week-end à Nice auprès de ses petits-enfants, fait une conférence à Paris et retourne à Copenhague en wagon-lit. Il entre à l'hôpital, deux jours après son retour à Bloomington, pour subir un quintuple pontage cardiaque réalisé grâce à $80 \mathrm{~cm}$ de veine prise dans sa jambe gauche; les os de la poitrine sont coupés, le cœur est arrêté plusieurs heures, puis il passe deux jours d'enfer en réanimation et deux jours de semi-enfer en soins intensifs ; le choc post-opératoire est pénible et, à la fin de l'année, il souffre toujours de sa jambe gauche. Néanmoins huit semaines après l'opération, le voilà en Mongolie où il n'est pas allé depuis douze ans; et pour alléger la longueur du voyage, il fait étape à l'aller à Francfort, au retour à Moscou, Wiesbaden et Bad Ems (le lieu de son service dans l'armée d'occupation en 1944-45). Et malgré sa santé déclinante puis l'opération et les voyages, il a eu le temps, durant cette même année 1982, de rédiger deux articles appelés à être des classiques, « Interpreters in Medieval Inner Asia » et « The Legendary Origins of the Türks », et de veiller à la construction d'un guesthouse dans sa propriété. Enfin il a la joie de voir paraitre à Budapest un volume, Tanulmányok, de quinze de ses articles publiés entre 1943 et 1977 et maintenant traduits en hongrois.

7 Deux ans plus tard, en 1984, en quatre mois et demi, entre mi-mars et fin juillet, il assiste à des colloques, entame des pourparlers et fait d'innombrables conférences d'abord à Seattle, puis en Hongrie, de retour à Bloomington, et aussitôt après à Taiwan, à Tokyo, à Pékin (où il se trouve pour la première fois), au Xinjiang, qu'il explore dans 
de difficiles conditions physiques, en Mongolie-Intérieure, à Tôkyô de nouveau, et à Budapest encore deux fois, en Allemagne, à Moscou, en Ouzbékistan et à Bakou, pour finir en famille à Nice. Lorsque, peu après, je lui rends visite à Bloomington, je le trouve frais, dispos, semblant n'avoir d'autre souci que d'organiser un mini-colloque et une réception pour moi. Mais son année n'est pas encore terminée: dans la seconde quinzaine d'octobre, il va en moto à Washington, D.C., un trajet de « 1300 miles (2145 $\mathrm{km}) »$.

8 Une des années les plus mouvementées fut, dit-il, 2003 où, à l'âge de 87 ans, il traversa six fois l'Atlantique en deux mois. Son voyage le plus extraordinaire fut, l'année suivante, sa découverte du pôle Nord sur un brise-glace nucléaire russe. Jusqu'aux dernières années de sa vie, son énergie paraît une bravade lancée à la raison. En 2004, au retour d'un voyage de $7600 \mathrm{~km}$ en voiture, il s'étonne de ne pas se sentir très bien, «juste une faiblesse générale peut-être justifiée par mon âge, 88 en quelques semaines ", m'écrit-il. Néanmoins, pour Noël 2006, il conduit sur les routes hivernales durant $1600 \mathrm{~km}$ afin de passer les fêtes avec son cher petit-fils Édouard et sa famille à Montréal ; puis à mi-mars 2007 il se lance sur un parcours de quelque $3900 \mathrm{~km}$ afin de participer à la réunion annuelle de l'American Oriental Society à San Antonio, alors que depuis un mois, il souffre de calculs rénaux; l'opération inévitable à son retour est suivie d'un arrêt du cœur et d'une pneumonie ; quand il rentre chez lui, à la mi-juin, il a perdu une quinzaine de kilos et n'a plus que la peau sur les os. Il doit alors se résigner à ne pas assister à la $50^{\mathrm{e}}$ PIAC qui se tient au Tatarstan, alors qu'il n'en a manqué aucune des précédentes, et il souffre profondément de ne pouvoir assister à sa remise d'un doctorat honoris causa de l'Université de Kazan. Puis, fin septembre, il est victime d'un méchant accident de voiture par la faute de l'adjoint du shérif; mais bien qu'il se remette mal de ses contusions, il repart pour Montréal au volant de la Cadillac qui a remplacé la Buick accidentée, pour passer de nouveau Noël chez son petit-fils. En mars 2008, un nouvel accident, au même carrefour que le précédent, détruit sa Cadillac qu'il remplace aussitôt. De sorte qu'en huit mois, il remarque qu'il a acheté une Buick et deux Cadillacs, "and thus contributed mightly to the prosperity of General Motors ». Cette année 2008, date de sa dernière lettre collective, est encore illuminée d'honneurs.

9 Notre amitié s'était renforcée en 1984 lors de mon passage chez les Sinor, dans leur sympathique maison située 5581 Lampkins Ridge, en pleine forêt, et dotée de ce joli pavillon indépendant qu'il venait de faire construire deux ans plus tôt : un guesthouse "self-sufficient" si besoin était, pour loger les invités. Jean, la troisième femme de Denis, était alors très prise par les examens qu'elle faisait passer à ses étudiants musicologues et le maître de maison était Denis seul, très affairé à nourrir et soigner les hôtes de la maison. La reine était Charlotte, une chienne tout en poils longs et fin museau, comme plus tard Fiona, qui lui succéda et, qui comme elle, accompagna Denis dans tous ses raids à moto ou en voiture. La photo de Fiona avait été communiquée à ses admirateurs potentiels par la carte de vœux accompagnant la lettre de Noël 2002, mais la chienne mourut le 21 octobre 2003 : «Some of you have met her, others knew her only from last year's Christmas card; few of you could know how closely our lives were interwoven. We traveled together, she came with me to my office, participated at committee-meetings, accompanied me on my visits with the Dean of the Chancellor. Her impeccable behavior gained her universal love and respect " (lettre de Christmas 2003). Toujours en 1984 le "zoo" de Lampkins Ridge, comme l'appelait Denis, comptait une chatte, Dorothy, selon moi mal aimée et doutant de tout et de tous; un adorable cochon d'Inde, Philip, roucoulant de tendresse; un exubérant mina («mainate» en 
français), genre de merle à collerette jaune, Tihany, dont les sifflements remplissaient la maison; enfin une perruche, Grüne, verte bien sûr, qui aimait faire sa gymnastique quotidienne sur le doigt de son dieu. Et tout alentour, régnait le bruissement des insectes. C'est cette maison que Sinor décrit dans son interview à Diogène en 2003, en réponse à la question "Qu'est-ce qui vous vient à l'esprit lorsque vous pensez à votre maison?» : «Ce qui me vient à l'esprit c'est ma maison dans les bois, pas un seul voisin en vue. Une maison que je n'ai à défendre ni de voleurs réels ni de terroristes imaginaires, mais des cerfs qui viennent grignoter mes fleurs et des ratons laveurs qui s'emparent de ma véranda, qui n'est qu'à quinze minutes en voiture d'une bibliothèque superbe » (Diogène, $\mathrm{n}^{\circ}$ 203, juillet-septembre 2003, p. 20). C'est cette maison que son propriétaire a donnée cette même année 2003, en octobre, à la Fondation de l'Université d'Indiana pour constituer un «Jean and Denis Sinor Faculty Fellowship in Central Eurasian History ».

Cependant, quelque intégré qu'il ait été dans la vie sociale américaine, il se sentait toujours profondément hongrois dans le fond de son cœur, me confiait-il. Alors qu'il est dénommé aux Etats-Unis «Deniss Saïnor ", son nom d'origine est Sinor Dénes (qui se prononce, avec un fort accent tonique sur les syllabes initiales, «Shinor Denesh ») ${ }^{4}$. Son lieu natal est un des joyaux que la Hongrie a perdu en 1918 et ne cesse de pleurer depuis lors: Kolozsvár, devenue Cluj, capitale de la Transylvanie dès lors roumaine, splendide ville qui vit naître Matthias Corvin, le fondateur de la royauté hongroise en 1458, et de grands hommes de lettres comme l'auteur de la trilogie transylvaine, Miklós Bánffy (1873-1950, brièvement ministre des Affaires étrangères en 1921-22). Sinor Dénes n'avait que deux ans lorsque la monarchie austro-hongroise s'effondra et que ses parents émigrèrent à Budapest, mais il garda toujours la nostalgie de Kolozsvár ${ }^{5}$. De bonne origine, il est alors élevé dans un établissement catholique dont il apprécie surtout les cours de latin et d'anglais et il passe ses vacances en Suisse où il améliore son allemand - langue de communication avec ses deux grands-mères - et son français. Lorsque le garçon a 16 ans, en 1932, sa famille s'installe dans le sud de la Hongrie, à Makó, où il poursuit ses études avec des tuteurs privés. Quand il s'inscrit à l'Université de Budapest, son intérêt premier est tourné vers le Moyen-Orient ancien; mais il est vite attiré par le prestige de deux altaiisants, Jules Nemeth (Nemeth Gyula 1890-1976), qui enseigne le turc depuis 1915, et Louis Ligeti (Ligeti Lajos 1901-1987), qui a créé une chaire de mongol jumelé au chinois en 1932, d'autant que la recherche d'ancêtres ethniques dans les steppes de l'Asie centrale est une préoccupation récurrente chez les intellectuels hongrois de ce temps ${ }^{6}$ : il n'y a ainsi pas à s'étonner que les études centreasiatiques aient pris, aux Etats-Unis, leur envol grâce à un Hongrois ${ }^{7}$ !

11 Comme on s'en doute Denis Sinor se révéla dès ses débuts un étudiant très doué, vite récompensé de nombreux prix. À l'automne de 1937, il gagna Berlin pour y bénéficier du bouillonnement encore visible les années précédentes dans le domaine des études orientales; mais la situation politique avait déjà contraint, ou allait bientôt contraindre, nombre d'orientalistes brillants à émigrer en raison d'une origine juive plus ou moins visible ou d'opinions politiques incorrectes : c'est ainsi que la sinologie française eut la chance de recevoir à ce moment Étienne Balazs (1905-1963) et Rolf Alfred Stein, sinologue et tibétologue (1911-1999) ${ }^{8}$. Le principal bénéfice que notre étudiant tira de son séjour à Berlin fut sa découverte des manuscrits en türk ancien rapportés par les expéditions allemandes à Turfan, qui lui fournirent le sujet de ses premiers articles ${ }^{9}$ et de sa thèse doctorale à son retour à Budapest. Cette thèse portant sur le bouddhisme des Türk anciens, bien que publiée en 1939 (en hongrois, dans Körösi 
Csoma Archivum, I) et primée, ne fut finalement jamais soutenue, et s'il fut un jour " docteur ", cela a été honoris causa, notamment de l'université de Szeged en 1971.

L'été 1939, il reçoit une bourse pour aller étudier à Paris avec le grand Paul Pelliot (1878-1945), le maître vénéré de Louis Ligeti. Il a alors 23 ans et ici commence une étape décisive de sa vie : il va tomber sous le charme de Paris, en dépit de la guerre qui éclate un mois après son arrivée, et le voilà, selon ses mots, devenu « un Hongrois parisien ». Bien plus tard, il aimera confesser que son grand bonheur est de promener son chien boulevard St-Michel. Durant la guerre, il suit les cours de Marcel Granet et de Paul Demiéville. Pour assurer sa vie matérielle - le montant de la bourse octroyée par le régent Horthy n'étant pas très élevé -, il occupe en 1939-40 un poste de répétiteur de hongrois à l'École des Langues orientales dite "Langues'O ", rue de Lille, auprès de la chaire d'Aurélien Sauvageot ${ }^{10}$, et en juin 1940, il se trouve momentanément être de facto directeur de l'Institut des Études hongroises, en l'absence du directeur en titre, Lipót/ Leopold Molnos ${ }^{11}$. En 1942, au moment où il va s'engager dans la Résistance, il est chargé d'une série de conférences à l'Institut des Hautes Études chinoises sur la protohistoire des peuples ouraliens et altaïques. Et il mentionne aussi, en passant, s'être occupé de commerce de fourrure pour survivre dans Paris occupé ${ }^{12}$.

Mais surtout, il suit, depuis la rentrée universitaire de 1939, les cours de Paul Pelliot au Collège de France ; et il réussit, grâce à son entregent, son charme et, disons-le tout sec, son culot, à être le seul des auditeurs et élèves du " Maître » - ainsi que celui-ci exigeait d'être appelé - qui ait jamais pu entretenir avec lui des rapports sociaux extra cathedram. Bien plus tard, devenu lui-même un maître vénéré, mais toujours plein d'humour, de gentillesse et de simplicité, il dépeindra par le menu le Pelliot qu'il a connu, et, bien loin de l'hagiographie habituelle sitôt que le nom de Pelliot est évoqué, il en démontrera les faiblesses de délicieuse (et cruelle) façon ${ }^{13}$. C'était «un grand mandarin", dit-il, qui n'acceptait une hiérarchie que s'il en occupait le sommet. « Kindness, friendliness at first sight, were not Pelliot's dominant traits of character ». "Modesty was not Pelliot's strong point : of it he had none. Accordingly, he would not tolerate contradiction and would dislike people who, in his view, could have intruded into what he considered his own territory ». Concernant sa méthode de travail : «In a not very respectful way I would describe his approach as leech-scholarship »; " he was unable, or unwilling to distinguish between the important and the unimportant ». Et curieusement : « he was less secure in Mongol, Turkic or Latin than in Chinese ».

Ces années de guerre vont enseigner à Sinor une leçon vitale, qui lui servira bien plus tard, lorsqu'en 1982 il subit une opération à cœur ouvert : il découvre qu'il n'a pas peur de la mort (il est de conviction catholique), alors qu'il la frôle souvent, d'abord engagé dans les Forces françaises de l'Intérieur ou FFI, où il est promu sergent major au $18^{\mathrm{e}}$ régiment de Dragons de l'armée du Rhin et Danube, puis incorporé avec son unité de résistance dans la $2^{\mathrm{e}} \mathrm{DB}$ du général Leclerc : une de ses bonnes histoires était celle de son entrée dans Strasbourg sur le deuxième tank qui délivra la ville. Il servit ensuite dans les troupes d'occupation d'une petite ville allemande, Bad Ems, avec le grade de maréchal-des logis-chef ${ }^{14}$; et c'est de là qu'en août 1945, il écrivit à Pelliot pour qu'il l'aide à obtenir sa démobilisation. Et Pelliot est semble-t-il intervenu, mais quand Sinor arrive à Paris, encore en uniforme, c'est pour saluer le cercueil du Maître le 31 octobre 1945. Suivent trois années difficiles pour Sinor, qui a obtenu la citoyenneté française pour faits de guerre, mais à qui, visiblement, l'establishment ne laisse pas la place qu'il mérite. Il enseigne alors le hongrois, le turc et le mandchou aux Langues'O. 
Il participe encore au XXIe Congrès international des Orientalistes qui se tient à Paris fin juillet 1948 (il a alors 32 ans); et il y présente une communication « Sur la légende de l'Oүuz qayan", où il montre le caractère bovin du héros mythique. Mais la rentrée universitaire le trouve à la faculté d'études orientales de la prestigieuse université de Cambridge : il fonde là l'enseignement du mongol classique, des études altaïques et, un peu plus tard, il y enseigne aussi le hongrois. A partir de 1955 jusqu'à son départ en 1962, il occupe le poste honorifique de secrétaire de la Royal Asiatic Society. Lorsque, au début des années soixante, son compatriote Thomas A. Sebeok (1920-2001) cherche à l'attirer dans le Uralic and Altaic Program qu'il a établi quelque temps auparavant à l'université d'Indiana, il pense ne pas répondre à l'invitation: «I saw no reason to exchange my post in arguably the best university in the world for that in a little-known university located in a land that was just recovering from McCarthyism. But I had a sabbatical coming up in Cambridge and I was not hostile to the idea of getting double salary for a semester. So I accepted a one-semester visiting professorship ", écrira-t-il plus tard ${ }^{15}$. Il prend son poste à la fin de 1961 et il est rapidement conquis : «I had the feeling that (contrary to the constant restraints experienced in Cambridge) they would "let me loose", giving me free rein to build what I was capable of building. I was also encouraged by the sympathetic understanding of my colleagues in the Program ${ }^{16}$. Il donne sa démission à Cambridge pour la rentrée universitaire de 1962 et il entame dès lors à Indiana University la carrière fulgurante que l'on connaît. Il parvient, entre autres, en pleine guerre froide à maintenir des rapports officiels cordiaux avec son pays natal.

S'il a quitté Paris en 1948, c'est certainement parce que ses talents n'y étaient par reconnus. Mais les mauvaises langues susurrent aussi qu'il a voulu, de la sorte, prendre ses distances avec un mariage manqué. En effet, il s'est marié en 1942 et, dans ses souvenirs sur Paul Pelliot, il mentionne la présence de sa femme auprès de lui lorsque, en octobre 1942, il passe en zone libre dans l'espoir de gagner l'Argentine où vivait son beau-père ${ }^{17}$. L'on sait tout juste que le mariage fut un échec et se termina vite par un divorce. D'un second mariage il eut une famille qui tint une grande place dans sa vie: une fille Sophie, qu'il fut heureux de retrouver après des années de séparation, et un fils prématurément disparu en 1972, mais qui lui a laissé une belle-fille française vivant à Nice, la très aimée Mylène, deux petits-fils Édouard et Manuel (ainsi, dit-on, qu'une petite-fille, Sarah, dont je dois avouer n'avoir jamais entendu parler). Édouard, qui était venu étudier à Indiana University en 1984, avant de fonder un foyer au Canada, est resté, jusqu'à la fin, très proche de son grand-père.

A partir de 1970, pour les amis de Denis, la maîtresse de maison qu'ils voient à ses côtés est la merveilleuse Jean. Jean avait 23 ans lorsqu'elle l'épouse en 1970 et devient plus hongroise que lui, si faire se peut : "She was the gardian of my family traditions. Her acquired knowledge of Hungarian, her love for that country, coupled with her fabulous memory made it that all the in-jokes of my childhood and adolescence lived on, that she could understand and use Hungarian literary allusions and was capable of enjoying the constant stream of idiocies that I can produce in any given period ", écrit-il à Noël 1999. Avec Jean, professeur de musique, spécialiste universellement reconnue de la musique et des méthodes de Kodály, enseignant autant à l'Université d'Indiana qu'à Budapest (en hongrois si je ne me trompe), la boucle de la vie de Denis Sinor se referme. C'est à Budapest, où elle se trouvait pour la réunion annuelle de l'International Kodály Organization dont elle était la présidente, lorsque le 9 mars 1999, elle meurt brutalement 
d'un arrêt cardiaque. Comme Sinor l'écrit dans un hommage funèbre : "She was fiftytwo, and we were in the twenty-ninth year of our fun-filled marriage. With her, there was never dull moment. She was a phenomenon whose activity brought the joy of music to thousands of children all over the world » et plus tard il ajoute à propos de sa mort : "She took away all my youth ». L'Académie des Sciences de Hongrie, dont Sinor était membre, se chargea de toutes les formalités administratives et Sinor put rapporter les cendres de sa femme pour les disperser dans le bois adjacent à sa maison. Soixante-cinq grosses caisses des papiers professionnels de Jean allèrent à l'Institut Kodály à Kecskemét.

Sinor annonçait, ces dernières années qu'il préparait son autobiographie: espérons qu'il est venu à bout de sa tâche et que nous aurons bientôt le récit exact d'une vie si extraordinairement ouverte sur le monde.

Photo du jeune Sinor durant son temps d'occupation militaire à Bad Ems en 1944-45

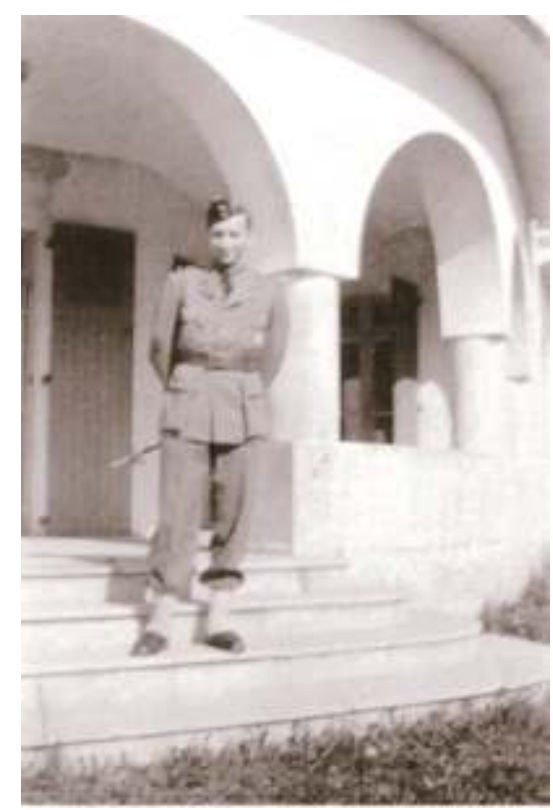

Cliché que nous devons à l'amabilité de M. Bernard Le Calloc'h, qui le publie par ailleurs dans son travail (sous presse) signalé supra note 11, Hetvenhárom év után Gróf Klebelsberg Kuno kivánsága beteljesedett Párizsban.

\section{NOTES}

1. On trouvera, sur Internet, dans SUDOC ou WorldCat, une liste des ouvrages dont Sinor a été soit l'auteur soit l'éditeur, sous son nom hongrois, Sinor Denes/Dénes.

2. Cf. la réimpression de ses articles d'histoire médiévale en deux volumes de Variorum Reprints: seize articles dans Inner Asia and its Contacts with Medieval Europe (Londres: Variorum Reprints, 1977) et dix-neuf articles dans Studies in Medieval Inner Asia (Aldershot: Ashgate, 'Variorum Collected Studies', 1997). 
3. J'ai appris, grâce à mon ami András Róna-Tas, que ce nom avait été suggéré par Sinor luimême, et qu'il l'avait choisi du fait que l'acronyme PIAC évoquait le terme hongrois piac signifiant "marché », la PIAC étant destinée à être un marché des connaissances dans le domaine de l'Eurasie centrale.

4. En hongrois, comme en chinois et en japonais, le nom de famille précède le nom personnel.

5. Je remercie András Róna-Tas pour ses informations sur les débuts de notre ami Sinor et Bernard Le Calloc'h pour des précisions sur son époque parisienne. Mais les interprétations que j'en ai pu tirer n'engagent que moi.

6. Sur l'ambiance intellectuelle tournée vers l'Asie intérieure dans la Hongrie du XIXe siècle et de la première moitié du XXe, voir ma notice biographique sur un autre orientaliste d'origine hongroise, Étienne Balazs (Balázs István, 1905-1963), dans Actualité d'Étienne Balazs (P.-É. Will \& I. Ang, eds, Collège de France/IHEC, 2010), particulièrement pp. 4-6 (note 18, corriger la référence à ma nécrologie de L. Ligeti en : Journal Asiatique, CCLXXVI, 1988).

7. On sait d'ailleurs qu'il a en plusieurs circonstances, à Paris, à Cambridge, enseigné le hongrois et publié une History of Hungary durant ses années britanniques, en 1959 (Londres: Allen \& Unwin).

8. Sur l'exode d'une foule de brillants esprits allemands, sinologues notamment, dans les années trente : Martin Kern, «The Emigration of German Sinologists 1933-1945 », Journal of the American Oriental Society, 118, 1990 : 507-529 ; David B. Honey, Incense at the Altar (New Haven : American Oriental Soc., 2001, p. 147 \& sq.

9. Il disait que son premier article avait été pour le T'oung Pao en 1937, édité par Pelliot, mais que c'était un travail médiocre à oublier (il s'agit de «Entwurf eines Erklärungsversuches der Pratītyasa mutpāda», TP, série II, XXXIII, pp. 381-394). Il narre de la sorte son premier contact avec Pelliot : «At the age of 19 I sent him [Pelliot] for publication an atrociously bad article, the receipt of which he never acknowledged, but which, for reasons unfathomable, he published in the T'oung Pao. In later years I never mustered the courage to ask him how this could happen ; I dreaded the thought that he might recognize in me the perpetrator of that sorry effort " (contribution de D. Sinor, « Remembering Paul Pelliot, 1878-1945 » dans Hartmut Walravens, éd., Paul Pelliot (1878-1945). His Life and Works - a Bibliography, Bloomington : Indiana Univ., 2001 : xxvxxxv, ou précédemment dans Journal of the American Oriental Society, 119, 1999, pp. 467-472).

10. Sur la période parisienne de la carrière de Sinor: Deux siècles d'histoire de l'École des langues orientales (Paris: Éditions Hervas, 1995, pp.186-187, notice par Bernard Le Calloc'h). Sur les ennuis d'Aurélien Sauvageot (1897-1988) durant la seconde guerre mondiale, op.cit. : 182-183; ou du même auteur, Az ismeretlen Sauvageot, a francia finnugorisztika atyja (Vac, 2010) : 35-54, biographie de Sauvageot en français, particulièrement pp. 44-46.

11. B. Le Calloc'h, Az ismeretlen Sauvageot (cité supra n. 9) : 45. Sur Molnos L. (1897-1982) : Deux siècles d'histoire : 186-187, notice par B. Le Calloc'h. Sur l'Institut hongrois de Paris, dit Collegium Hungaricum, et le bref passage de Denis Sinor à sa tête : B. Le Calloc'h, « Léopold Molnos, pionnier des relations culturelles franco-hongroises ", Association des anciens élèves de l'INALCO, Le Bulletin, oct. 1998: 81-88, notamment 86 ou, plus fouillé, Hetvenhárom év után Gróf Klebelsberg Kuno kivánsága beteljesedett Párizsban [Après 73 ans, le rêve du Comte Kuno Klebelsberg a enfin été réalisé à Paris], sous presse dans les Cahiers de la Fondation Klebelsberg (particulièrement pp. 18-24).

12. D. Sinor, « Remembering Paul Pelliot » (cité supra n. 9), dans l'éd. due à H. Walravens : xxxii, ou dans JAOS : 470.

13. Op.cit.

14. Id.

15. Inner Asian \& Uralic National Resource Center, Newsletter, Winter 2003, "A Word from the Director".

16. Id. 
17. «Remembering Paul Pelliot» : xxxii. Selon B. Le Calloc'h (communication personnelle du 22 juilet 2011), il a épousé Jacqueline du Faucon le 22 septembre 1942, en pays basque à Guétary. 